\title{
Émotion, ipséité, liberté : Réflexions à propos des fondements de la théorie sartrienne des émotions
}

\author{
Par FABIO RECCHIA
}

Université de Liège

Résumé Entretenue durant la drôle de guerre, la correspondance épistolaire entre De Beauvoir et Sartre révèle une insatisfaction de ce dernier à l'égard de sa théorie des émotions. Celle-ci manquerait en effet de fondements solides. Répondant à ce constat, nous tenterons de dégager les fondations de cette théorie de manière à expliciter ses enjeux. Nous replacerons, pour ce faire, l'Esquisse d'une théorie des émotions dans la perspective plus générale de la théorie de l'intentionnalité du premier Sartre. De la sorte nous montrerons, d'une part, comment ce texte renouvelle significativement la catégorie de l'action en concevant l'émotion comme une activité ; et nous examinerons, d'autre part, l'interaction existant entre l'émotion et l'ipséité de la conscience. Cette discussion dévoilera ainsi l'intentionnalité émotive comme une passion active reconduisant au fondement de la conscience qui est sa liberté personnelle.

S'inscrivant dans l'engouement pour l'Esquisse d'une théorie des émotions ${ }^{1}$ suscité notamment par la parution du dernier volume des Études sartriennes qui invite à lire ce texte de 1939 autrement que « comme on lit un brouillon $»^{2}$, cet article interroge la cohérence et la pertinence de l'Esquisse dans l'économie générale de la production philosophique du premier Sartre.

\footnotetext{
${ }^{1}$ J.-P. Sartre, Esquisse d'une théorie des émotions, Paris, Hermann, 1995 (désormais cité «ETE»).

${ }^{2}$ F. Caeymaex \& G. Cormann, « Présentation », dans F. Caeymaex, G. Cormann \& V. de Coorebyter (dir.), Etudes sartriennes, Bruxelles, Ousia, 2014, vol. 17-18 : Repenser l'Esquisse d'une théorie des émotions, p. 4.
} 
En effet, ce n'est pas sans motif que le lecteur peut entretenir quelques soupçons quant à la qualité de ce texte. Bien avant que plusieurs commentateurs n'identifient les diverses opacités de l'Esquisse, Sartre lui-même, quelques mois après la parution de son ouvrage, confessait à Simone de Beauvoir son inquiétude. "La théorie », écrivait-il le 3 mars 1940, «est montrée mais non point démontrée. La préface est ce qu'il y a de mieux $»^{1}$. En d'autres termes, la défectuosité de l'Esquisse naîtrait de ce que son auteur présente une théorie des émotions sans prendre le soin d'expliciter, sinon d'élaborer, les fondements sur lesquels s'appuie son discours. Cela justifierait, en conséquence, l'habitude de lire l'Esquisse comme un brouillon ou, disons, une introduction fort commode aux grands boulevards de la pensée sartrienne.

Aussi, si notre problème est de lire l'Esquisse, dans cet article, notre objectif sera en première intention de dégager les fondations de la théorie sartrienne des émotions, de manière à ce que nous puissions expliciter ensuite un certain nombre de ses enjeux. À cette fin, notre stratégie de lecture consistera à resituer l'Esquisse dans la perspective plus générale de la théorie sartrienne de l'intentionnalité. En d'autres mots, pour mettre en exergue les fondements et les enjeux du texte de 1939, nous décrirons les spécificités de l'intentionnalité émotive. Au lieu, donc, de mobiliser les sciences humaines ${ }^{2}$, l'histoire de la phénoménologie ${ }^{3}$ ou l'histoire de la philosophie ${ }^{4}$ elle-même pour éclairer l'Esquisse, nous lirons plutôt Sartre à partir de Sartre. Compte tenu de ce que notre méthodologie rapproche le concept d'émotion de celui d'intentionnalité, nous commenterons l'ouvrage de 1939 à partir de « l'article

\footnotetext{
${ }^{1}$ J.-P. Sartre, Lettres au Castor et à quelques autres, Paris, Gallimard, 1983, tome 2, p. 115.

${ }^{2} C f$. J. Englebert, «La magie et la sorcellerie des visages comme socle anthropologique de la philosophie sartrienne», dans F. Caeymaex, G. Cormann \& V. de Coorebyter (dir.), Études sartriennes, op. cit., p. 41-59; H. Oulc'hen, «L'émotion dans la pratique : de Sartre à Bourdieu », dans F. Caeymaex, G. Cormann \& V. de Coorebyter (dir.), Études sartriennes, op. cit., p. 169-198.

${ }^{3}$ Cf. V. de Coorebyter, Sartre face à la phénoménologie, Bruxelles, Ousia, 2000, Ch. 4, p. 122-158 ; A. Zincq, "Corporéité et émotion dans les phénoménologies asubjectives de Sartre et de Patočka », dans Études sartriennes, op. cit., p. 139-168.

${ }^{4} C f$. R. Breeur, "Passion à volonté », dans Bulletin d'analyse phénoménologique VII 3 (2006), p. 3-21 ; G. Cormann, «Passion et liberté. Le programme phénoménologique de Sartre », dans P. Cabestan \& J.-P. Zarader (dir.), Lectures de Sartre, Paris, Ellipses, 2011, p. 93-115.
} 
sur l'intentionnalité ${ }^{1}{ }^{1}$ chez Husserl ainsi qu'avec différents passages de L'Être et le Néant ${ }^{2}$, plutôt que de lire l'Esquisse à partir de La Transcendance de l'Ego ou de L'Imaginaire. De manière plus précise, ce travail d'analyse textuelle s'appuiera sur trois segments de la philosophie sartrienne : nous examinerons la troisième partie de l'Esquisse d'une théorie des émotions ${ }^{3}$ en mobilisant les premières analyses que Sartre formule en 1934 à propos du principe d'intentionnalité ${ }^{4}$; le début de la quatrième partie de L'Etre et le Néant, où le texte fait allusion à la théorie des émotions de $1939^{5}$; et, enfin, plus largement, les descriptions des structures immédiates du pour-soi qui, bien qu'elles se situent dans la seconde partie de l'ontologie phénoménologique ${ }^{6}$, seront lues comme les conséquences directes de la preuve ontologique figurant au début de $1^{\prime}$ Essai de $1943^{7}$. Partant ainsi de cette intertextualité, notre texte prendra concrètement la forme d'une analyse phénoménologique de l'intentionnalité émotive que nous approfondirons en quatre étapes.

Pour nous donner un cadre discursif à partir duquel analyser adéquatement l'émotion, nous expliciterons tout d'abord la polysémie et les usages du principe sartrien d'intentionnalité, en rapprochant l'article de 1934 de l'Essai de 1943 (section 1). Nous décrirons ensuite l'intentionnalité émotive, au croisement de l'Esquisse et de L'Etre et le Néant; cela permettra de dégager les fondations ainsi que la cohérence générale de la théorie sartrienne des émotions (section 2). Après quoi, nous identifierons un premier enjeu du texte de 1939 en indiquant de quelle manière cet ouvrage conçoit l'émotion comme une activité et contribue à élargir, comme L'Etre et le Néant l'accomplira, l'extension de la catégorie de l'action (section 3). Nous examinerons enfin le processus de stabilisation du circuit de l'ipséité qu'assurent les émotions, en espérant par là même dégager un deuxième enjeu philo-

${ }^{1}$ J.-P. Sartre, « Une idée fondamentale de la phénoménologie de Husserl : l'intentionnalité », dans Id., La Transcendance de l'Ego et autres textes phénoménologiques, Paris, Vrin, 2003, p. 87-89 (désormais cité «I »).

2 J.-P. Sartre, L'Être et le Néant, Paris, Gallimard, Coll. « Tel », 1976 (désormais cité « $E N »)$.

${ }^{3}$ Cf. ETE, p. 69-117.

${ }^{4} C f$. I, p. 88-89. Nous considérons en effet que l'article sur l'intentionnalité a été rédigé en 1934 puis publié en 1939 (Cf. V. de Coorebyter, Introduction, dans J.P. Sartre, La Transcendance de l'Ego et autres textes phénoménologiques, Paris, Vrin, 2003, p. 8-12).

${ }^{5}$ Cf. $E N$, p. $477-497$

${ }^{6}$ Cf. $E N$, p. $109-141$.

${ }^{7}$ Cf. EN, p. 26-29. 
sophique pour le moins fondamental et cependant fort peu explicite dans le texte de Sartre (section 4). Plus fondamentalement encore, en fondant ses analyses sur une lecture polyphonique du texte de Sartre où nous mettrons l'accent sur la polysémie des concepts ainsi que sur un travail de description intense de l'intentionnalité, notre texte espère contribuer à éclairer les bases, la logique immanente, la fonction et la nécessité de la théorie sartrienne des émotions.

\section{Polysémie et usage du principe sartrien d'intentionnalité}

Afin de localiser les fondations de la théorie sartrienne de l'intentionnalité émotive, repartons de l'article sur l'intentionnalité chez Husserl à partir duquel on peut dégager les grands axes de la production philosophique du premier Sartre. Car, comme l'a remarqué Jean Hyppolite, cet article de 1934 s'apparente moins à une présentation fidèle de la pensée de Husserl qu'à une « subversion » ${ }^{1}$ de celle-ci, au terme de laquelle Sartre ébauche quelques-uns des grands thèmes d'une phénoménologie « originale $»^{2}$. En effet, si Sartre admet avec Husserl le principe d'intentionnalité selon lequel «toute conscience est conscience de quelque chose $»^{3}$, il précise néanmoins que ce « quelque chose » est extérieur à la conscience ${ }^{4}$. Aussi toute conscience estelle consciente de quelque chose qui n'est pas elle. Selon Sartre, l'intentionnalité est une relation directe à cette extériorité, plus qu'elle ne désigne une propriété des actes mentaux. En outre, puisque la conscience s'identifie à cette visée intentionnelle, définie à l'instant comme une relation à une extériorité extra-mentale, la conscience «n'a pas de dedans $»^{5}$; elle est vide $^{6}$. Par conséquent, la phénoménologie, qui produit le compte-rendu descriptif de cette relation, a pour objet d'analyse un rapport reliant une conscience vide à un monde. Sartre baptise ce rapport du nom d'existence ${ }^{7}$ et, de là, élabore une phénoménologie réaliste en rupture par rapport au phénoménalisme husserlien.

Ainsi la conception relationnelle de l'intentionnalité défendue par l'article de 1934 a pour conséquence la transphénoménalité d'un être réel par

${ }^{1}$ J. Hyppolite, Figures de la pensée philosophique, Paris, P.U.F., 1972, p. 759.

${ }^{2}$ V. de Coorebyter, Introduction, op. cit., p. 21.

${ }^{3}$ I, p. 89.

${ }_{5}^{4} I d$.

${ }^{5}$ I, p. 88.

${ }^{6}$ I, p. 89.

${ }^{7}$ Id. 
rapport à une conscience vide et, plus globalement, la fondation d'un existentialisme phénoménologique où la conscience se rapporte à un monde qui lui préexiste. En ce sens, ce texte semble ébaucher une idée maitresse de L'Etre et le Néant : la «preuve ontologique ». En effet, en décrivant l'intentionnalité comme un lien entre une conscience et son monde, l'article de 1934 détient virtuellement cette idée directrice de l'ontologie phénoménologique de 1943 : l'intentionnalité est une relation entre deux êtres de telle manière que l'existence de l'un donne la justification de la présence de l'autre. En d'autres termes, puisque dès 1934, la conscience est « conscience d'autre chose que soi » ${ }^{1}$, elle assure la révélation d'une " chose » étrangère dont elle «souffre d'abord la qualité objective $»^{2}$. Aussi, la relation intentionnelle, que l'article berlinois place au cœur de la conscience, peutelle en 1943 « servir de "preuve ontologique" d'un [être]-en-soi» ${ }^{3}$ transphénoménal, au sens où elle renvoie à un être qui « se donne comme existant déjà lorsqu'elle le révèle ${ }^{4}$. Bien plus, l'intentionnalité assure une liaison entre deux «régions d'être $»^{5}$ : car si l'en-soi désigne l'être révélé par la conscience intentionnelle, cette dernière peut être également thématisée en termes ontologiques dans la mesure où elle s'identifie à "l'être du sujet » ${ }^{6}$ que Sartre appelle «l'être-pour-soi». Et tout l'effort de l'ontologie phénoménologique consiste à soutenir avec force que le pour-soi est pourvu d'un «néant interne ${ }^{7}$. De sorte qu'au lieu d'être vide comme en 1934, la conscience de 1943 est un néant empêchant toute coïncidence du sujet avec lui-même.

Loin de se réduire à une question de mots sur la manière dont il faut qualifier la béance intrinsèque de la conscience, ce nouvel usage de la terminologie traduit en réalité une évolution conceptuelle de l'œuvre de Sartre, de sorte que les textes de 1934 et 1943 développent des théories différentes de la conscience. Et de l'écart formé entre ces deux conceptions, motivé sans doute par l'écriture des Carnets de la drôle de guerre ${ }^{8}$, découle une sophis-

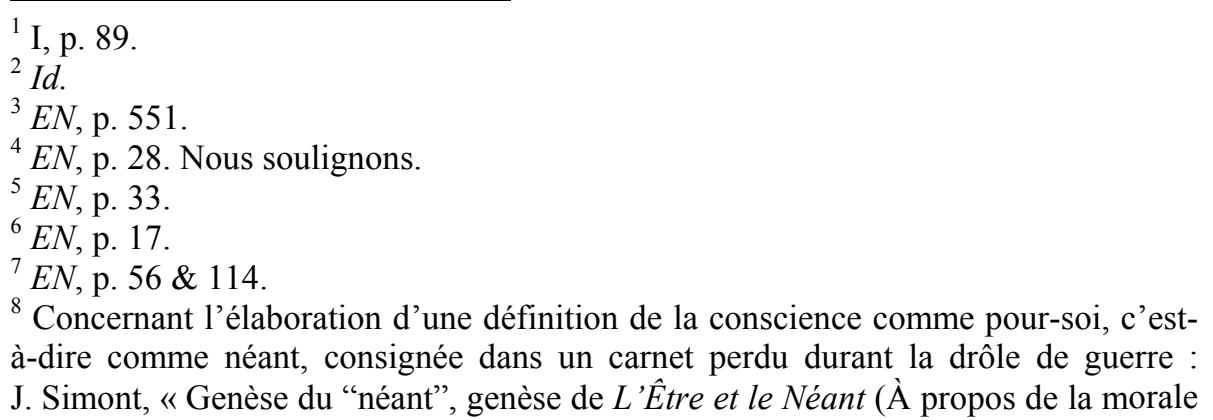


tication de la théorie sartrienne de l'intentionnalité. En effet, là où l'article de 1934 indiquait que la conscience est vide parce qu'elle est intentionnelle, l'essai d'ontologie phénoménologique, en soulignant que « le rapport interne du pour-soi à l'en-soi [...] tire son origine de l'insuffisance d'être du poursoi ${ }^{1}$, soutient que la conscience est intentionnelle car elle est d'abord fissurée par un néant interne. Cela n'est pas sans incidence sur la nature même du phénomène d'intentionnalité dont l'ontologie phénoménologique va produire une description différente du texte de 1934. Car en trouvant son origine dans une négation interne au cœur de la conscience, l'intentionnalité devient une puissance de négation. De manière plus précise, Sartre décrit cette puissance comme l'élan par lequel le pour-soi «se jette vers sa fin $»^{2}$. Si bien que le principe d'intentionnalité de 1943 désigne, non seulement une négation du donné transphénoménal, mais aussi un projet intentionnel d'un possible à partir d'un état de choses réel ${ }^{3}$.

En outre, alors que l'ontologie phénoménologique construit progressivement une polysémie du concept d'intentionnalité en définissant la relation intentionnelle, tantôt comme une puissance, tantôt comme un projet, Sartre va faire usage de ce principe pour lester la conscience d'une structure identitaire ${ }^{4}$. Comme le remarque en effet $L$ 'Etre et le Néant, « le possible est [...] une structure du pour-soi $»^{5}$ en ce sens que le possible est un manque du

et de l'ontologie de Sartre) », dans J.-M. Mouillie \& J.-P. Narboux (dir.), Sartre. L'Être et le néant, nouvelles lectures, Paris, Les Belles Lettres, 2015, p. 35-56.

${ }^{1} E N$, p. 634.

${ }^{2} E N$, p. 602.

${ }^{3} E N$, p. 528.

${ }^{4}$ Lorsque nous affirmons que Sartre mobilise l'intentionnalité de la conscience pour y élaborer une «structure identitaire», nous voulons dire qu'il emploie ce concept afin de poser que l'identité du pour-soi se constitue à partir de processus intentionnels. Cette idée remonte, selon nous, à La Transcendance de l'Ego, où Sartre justifie l'unité et l'individualité de la conscience à partir de son « jeu d'intentionnalités » (J.P. Sartre, La Transcendance de l'Ego, Paris, Vrin, 2000, p. 22 ; désormais cité « $T E »)$. D'où il suit que l'identité, ou la singularité, de la conscience est rendue possible par ses intentionnalités. Elle est, en d'autres termes, charpentée dans des processus intentionnel d'identification. Approfondissant cette thèse, L'Être et le Néant indiquera ensuite que ces processus consistent à articuler les vécus de la conscience autour d'un rapport à soi comme possible ; c'est-à-dire autour de ce que Sartre appelle un "circuit de l'ipséité ». Pour un examen complet des différentes facettes du problème de l'identité chez Sartre, nous renvoyons à la référence suivante : J.-M. Mouillie, Sartre. Conscience, Ego et psyché, Paris, P.U.F., 2000.

${ }^{5}$ EN, p. 33. 
pour-soi. Par conséquent, au lieu qu'elle se tienne en face de ce possible qu'elle doit réaliser, la conscience s'identifie à ce possible qu'elle pose dans son projet comme son manque ${ }^{1}$. C'est dès lors elle-même, par l'entremise du monde, que la conscience atteint dans son projet. C'est pourquoi Sartre appelle

« circuit de l'ipséité » le rapport du pour-soi avec le possible qu'il est - et «monde» la totalité de l'être en tant qu'elle est traversée par le circuit de l'ipséité ${ }^{2}$.

Pour le dire une première fois, le circuit de l'ipséité désigne le modèle que propose l'essai de 1943 pour penser l'identité personnelle du pour-soi comme un équilibre dynamique auquel nous verrons l'émotion participer. Pour l'heure, contentons-nous de deux remarques. Premièrement, l'ontologie phénoménologique joint à l'ipséité un circuit de significations grâce à un usage habile du principe d'intentionnalité. En effet, lorsqu'il réalise un possible à partir d'ustensiles, le projet intentionnel assigne simultanément une valeur sémantique à chaque ustensile en fonction de la manière dont il conduit au possible ${ }^{3}$. Deuxièmement, puisque «l'être dit libre est celui qui peut réaliser ses projets ${ }^{4}$, la réalisation d'une possibilité projetée est donc l'acte qui relie, à son tour, le circuit de l'ipséité de la conscience à sa liberté. De manière plus précise, la réalisation d'un projet qui correspond à la concrétisation de la liberté au travers d'une conduite, se produit de deux manières : "soit par la volonté » qui construit des chaines d'ustensiles permettant de parvenir au possible, «soit par des efforts passionnels $»^{5}$, c'est-à-dire des émotions qui désarment la signification associée aux ustensiles $^{6}$. Dans l'Essai de 1943, tout se passe donc comme si un réseau secondaire d'intentionnalités définissait un ensemble de manières de réaliser volontairement ou émotionnellement un seul et même projet du pour-soi ${ }^{7}$ tentant de suppléer son manque d'être originel. Néanmoins, qu'elle soit une volonté ou un effort passionnel, la mécanique intentionnelle qui découle de ce néant d'être reste toujours décrite par Sartre comme une conduite qui est l'expression de la liberté humaine.

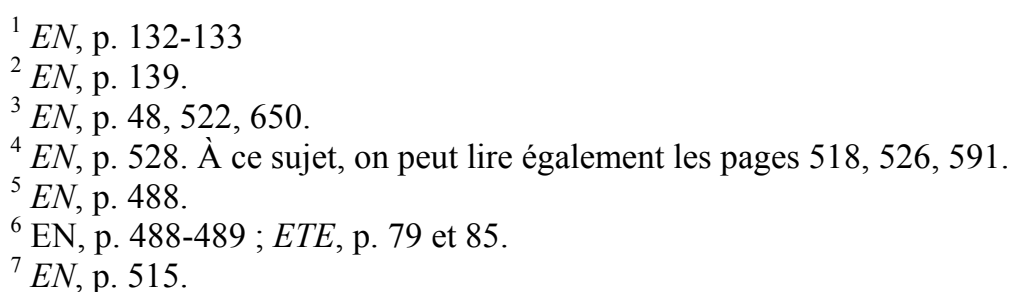


Aussi l'examen que l'on vient de mener permet-il de mettre en évidence que, dans l'ontologie phénoménologique, le concept d'intentionnalité recouvre toujours trois significations. $1^{\circ}$ La relation intentionnelle est une puissance de négation de l'en-soi transphénoménal dont le néant interne de la conscience est la cause. $2^{\circ}$ Elle est le projet d'un possible à partir duquel apparaît un monde signifiant. $3^{\circ}$ Elle est une conduite au travers de laquelle le pour-soi réalise sa liberté. Par ailleurs nous avons montré que Sartre adosse à cette polysémie un usage original de la relation intentionnelle. Car il l'emploie pour lester la conscience d'une structure identitaire qui est le circuit de l'ipséité du pour-soi. C'est à partir de cette compréhension élargie de l'intentionnalité sartrienne que l'on va maintenant dégager les fondations et les enjeux de la théorie sartrienne des émotions.

\section{L'intentionnalité en tant qu'elle est émotive}

D'après Sartre, l'émotion n'est pas un « orage physiologique ${ }^{1}$, ni même un accident ${ }^{2}$, mais une « réponse adaptée $»^{3}$ à certaines situations. Alors que le pour-soi réalise factuellement son projet dans le monde, il arrive en effet que ce dernier oppose une résistance aux conduites volontaires de la conscience. Aussi « lorsque les chemins tracés deviennent trop difficiles ou lorsque nous ne voyons pas de chemin » ${ }^{4}$, l'émotion intervient comme une «alternative spontanée à l'action pratique $»^{5}$, indique V. de Coorebyter, et elle produit

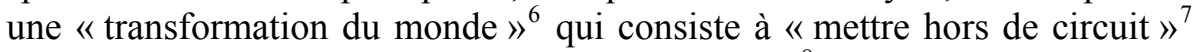
les éléments opposant un " coefficient d'adversité » ${ }^{8}$ à la conduite volontaire. Toutefois, au lieu, par exemple, de déplacer ou de briser cet existant, la conscience émotive 《 modifie son rapport avec cet être ${ }^{9}$ afin de le transformer. C'est pourquoi, la conduite émotive

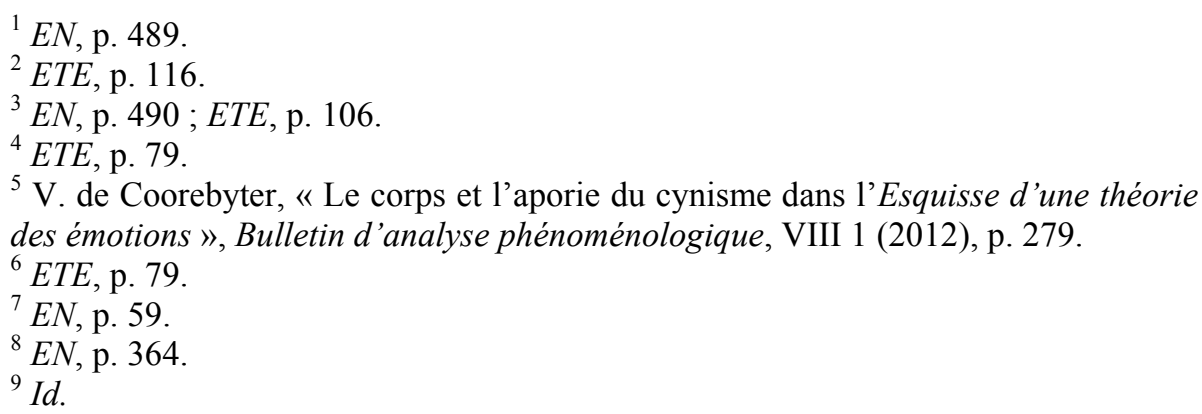


n'a pas pour fin d'agir réellement sur l'objet en tant que tel par l'entremise de moyens particuliers. Elle cherche à conférer à l'objet par elle-même, et sans le modifier dans sa structure réelle, une autre qualité, une moindre existence ${ }^{1}$.

Plus globalement, puisque l'émotion supprime les qualités contraignantes des objets mondains, la conduite passionnelle est « une dégradation spontanée et vécue de la conscience face au monde $»^{2}$ qui précipite cette dernière dans un «monde magique ${ }^{3}$. Car la conduite passionnelle consiste à faire « comme si les rapports entre des choses à des potentialités n'étaient pas réglés par des processus déterministes $»^{4}$. Ainsi, en neutralisant les qualités problématiques des choses au moyen d'une révision du rapport qui lie ces dernières à des possibles, l'émotion assure une réponse adaptée à une situation difficile. Si bien qu'elle « peut servir de remplacement $»^{5}$ à la conduite volontaire. Mieux encore, en décrivant les émotions comme des conduites passionnelles qui produisent des «assouvissements symboliques de nos désirs ${ }^{6}$ et de nos projets, l'ontologie de 1943 soutient que les passions sont, au même titre que les volitions, "certaines attitudes subjectives par lesquelles nous tentons d'atteindre aux fins posées par la liberté originelle » ${ }^{7}$.

Néanmoins, cette description des propriétés de l'intentionnalité émotive pose question car elle semble reposer sur des propositions contradictoires entre elles. Le texte de 1939 pose au moins quatre problèmes. a) L'émotion y est définie comme un rapport de la conscience au monde et comme la négation de ce rapport, dans la mesure où elle est une dégradation du lien existant entre la conscience et le monde. b) L'intentionnalité émotive est une constitution magique du monde, alors qu'elle est par avance une dégradation du lien existant entre la conscience et son monde. c) Dans $L$ 'Etre et le Néant, la passion est certes une conduite qui parvient aux fins posées par le projet mais, dans l'Esquisse, elle met «en veilleuse ${ }^{8}$ la conscience et neutralise, donc, le projet d'une fin qui la définit comme conscience. d) Enfin l'émotion est une manière d'agir lorsque toutes les routes sont barrées, mais elle n'est pas une manière d'agir effective.

${ }^{1}$ ETE, p. 81

${ }^{2} E T E$, p. 100.

${ }^{3}$ ETE, p. 115.

${ }^{4}$ ETE, p. 79.

${ }^{5}$ ETE, p. 82.

${ }^{6} E N$, p. 489.

${ }^{7} E N$, p. 488.

${ }^{8}$ ETE, p. 87. 
À dire vrai, comme le remarque A. Hervy, « les passages de l'ouvrage de 1939 sont nombreux où l'on hésite $»^{1}$, car l'addition de ces « paradoxes » ${ }^{2}$ suscite, sinon un «malaise ${ }^{3}$, du moins un doute quant à la cohérence générale de cet écrit. Toutefois, il nous semble que l'opacité du texte tient moins à une «maladresse ${ }^{4}$ de son auteur qu'à la polysémie du concept d'intentionnalité émotive, qui reste inaperçue en 1939. Comme s'il s'agissait d'une seule et même chose, l'émotion est en effet décrite comme une réponse, une transformation et une conduite. Aussi, si l'on veut dissiper cette confusion, il faut décrire les spécificités de l'émotion en les rapportant aux propriétés générales de l'intentionnalité qui ont été dégagées plus haut. Cela nous permettra de mieux discerner les distinctions subtiles et les niveaux de complexités de la théorie des émotions ; mais aussi, et surtout, de cibler son niveau le plus profond qui est celui de sa fondation dans le néant interne du pour-soi, c'est-à-dire dans la liberté de la conscience.

Si l'on admet avec L'Être et le Néant que l'intentionnalité désigne une puissance de négation, l'émotion peut être à son tour décrite à l'instar de ce que F. Caeymaex décrit comme un «travail du négatif » ${ }^{5}$. Que l'émotion soit une réponse, cela signifie alors qu'elle est un pouvoir de dire non à l'adversité opposée par l'en-soi transphénoménal. À dire vrai, il semble même que ce soit contre elle-même que la conscience émue exerce son pouvoir de négation. En effet, la conscience émotive met «hors de circuit un existant particulier 》 en se mettant «elle-même hors de circuit par rapport à cet existant ». En d'autres termes, elle modifie « son rapport avec cet être $»^{6}$ qui la définit comme conscience. De telle sorte que l'émotion est une puissance de négation intransitive et, plus globalement encore, selon l'interprétation formulée par G. Cormann et J. Englebert, une « expérience que la conscience fait de sa négativité ${ }^{7}$, c'est-à-dire une expérience de sa propre dégradation dont la cause est une négation émise par le pour-soi à sa propre adresse.

\footnotetext{
${ }^{1}$ A. Hervy, "Émotion et aliénation», dans F. Caeymaex, G. Cormann \& V. de Coorebyter (dir.), Études sartriennes, op. cit., p. 21.

${ }^{2}$ A. Hervy, «Émotion et aliénation », art. cit., p. 22.

${ }^{3}$ V. de Coorebyter, « Le corps et l'aporie du cynisme », art. cit., p. 276.

${ }^{4}$ Ibid., p. 273.

${ }^{5}$ F. Caeymaex, « Vie et praxis : le statut de l'organisme dans la Critique de la raison dialectique », Bulletin d'analyse phénoménologique VI 2 (2010), p. 158.

${ }^{6}$ EN, p. 59.

${ }^{7}$ G. Cormann, « Émotion et réalité chez Sartre, Remarques à propos d'une anthropologie philosophique originale ", dans Bulletin d'analyse phénoménologique VIII 1 (2012), p. 297.
} 
Si l'on soutient avec le texte de 1943 que l'intentionnalité est le nom donné au projet intentionnel d'un possible, on peut ainsi justifier le «caractère étrangement finaliste $»^{1}$ de certaines formulations de l'ouvrage de 1939. Que la conscience émotive soit une dégradation, cela signifie en effet que la conscience transforme son rapport au monde en projetant des " possibles inédits » ${ }^{2}$ qui assurent «la saisie de rapports nouveaux et d'exigences nouvelles $»^{3}$. De manière plus précise, la transformation de la conscience émotive coïncide avec la constitution sémantique d'un monde dont le sens, dit A. Hervy, est régi par les « lois de la magie » ${ }^{4}$. En ce sens, l'intentionnalité émotive peut être décrite comme le projet d'une transformation magique du monde par laquelle ses possibilités sont absolutisées. En effet, grâce au corps qui assure le «moyen d'incantation ${ }^{5}$ de cette magie, le projet émotif «désarme » ${ }^{6}$ l'adversité d'une situation en exagérant ses potentialités. Sans que rien ne change effectivement à l'intérieur d'une situation, une grappe de raisin devient ainsi trop verte ${ }^{7}$, une difficulté soudainement trop difficile, un danger possiblement trop dangereux. Si bien que la conscience se détourne de cette grappe, évite de se heurter à cette difficulté, esquive par avance ce danger, mais désamorce dans tous les cas les contraintes qui font opposition à ses projets, puisqu'elle ne s'y confronte pas.

Enfin, si l'on affirme avec l'essai d'ontologie phénoménologique que l'émotion est une conduite par laquelle le pour-soi assouvit ses projets, on peut remarquer avec G. Cormann que «le problème de la liberté [...] traverse l'Esquisse d'une théorie des émotions $»^{8}$. Dans L'Être et le Néant, la conduite passionnelle est identifiée à une concrétisation de la liberté du poursoi car l'émotion y est définie comme une « manière particulière ${ }^{9}$ pour lui de réaliser son possible. Sartre décrit cette réalisation comme un assouvissement symbolique. En effet, là où la conduite volontaire réalise un possible en le matérialisant au travers d'une chaine d'ustensiles, l'émotion procède à un travail sur la signification associé aux ustensiles. Elle réalise le possible du

\footnotetext{
${ }^{1}$ V. de Coorebyter, « Le corps et l'aporie du cynisme », art. cit., p. 278.

${ }^{2}$ R. Gély, «Émotion, imagination, incarnation: Réflexion à partir de l'Esquisse d'une théorie des émotions » dans Bulletin d'analyse phénoménologique VIII 1 (2012), p. 344.

${ }^{3}$ ETE, p. 79.

${ }^{4}$ A. Hervy, «Émotion et aliénation », art. cit., p. 25.

${ }^{5}$ ETE, p. 93.

${ }^{6}$ ETE, p. 113.

${ }^{7}$ ETE, p. 82.

${ }^{8}$ G. Cormann, « Passion et liberté », art. cit., p. 115.

${ }^{9}$ EN, p. 490.
} 
pour-soi en transformant la signification de l'en-soi. Et cette transformation consiste à pratiquer une exagération magique du sens des ustensiles. D'après nous, cette exagération peut prendre deux formes différentes.

Dans le premier cas, l'émotion exagère le sens d'un ustensile pour amoindrir la « distance » qui le sépare du possible à réaliser. Une fois cet écart consumé, l'émotion établit un rapport d'identité entre le possible à réaliser et l'ustensile qui permet sa réalisation. Elle confond ainsi la fin et le moyen, entrainant dès lors la réalisation du possible puisque le possible devient un état de choses réel. La joie sera par exemple une conduite magique permettant «la possession de l'objet désiré comme une totalité instantanée $»^{1}$.

Dans le second cas, l'émotion exagère le sens d'un ustensile pour accroitre la distance qui le sépare du possible à réaliser. Un fois cet écart maximalisé, l'émotion établit un rapport d'antagonisme entre le possible à réaliser et l'ustensile qui permet sa réalisation. Elle rompt ainsi le lien existant entre la fin et le moyen. Ici, la tristesse sera la conduite magique permettant de " mettre en veilleuse $»^{2}$ un univers d'outils. Toutefois, il faut remarquer que la tristesse et, plus globalement, les conduites magiques qui lui sont apparentées, opèrent bien la réalisation d'un possible. Certes, en interrompant la liaison entre le possible et le réel, l'émotion reporte la réalisation du possible à une situation ultérieure. Néanmoins, c'est à l'intérieur même de la situation présente que la conscience émotive se donne la garantie d'une réalisation future. De sorte que la conscience est déjà en train de réaliser son possible, au moment même où elle se convainc qu'elle ne le peut pas, parce qu'elle pose implicitement qu'elle pourrait le faire dans une autre situation.

Néanmoins, il est clair que l'une et l'autre forme de réalisation passionnelle du possible placent la conscience dans un état pour le moins troublant. Tantôt, l'émotion contraint la conscience à identifier l'état de choses réel à l'état de chose possible ; tantôt elle noue un rapport ambigu entre le futur et le présent. De sorte que le pour-soi se rapporte au monde à partir de catégories flottantes, concrétisant dès lors sa liberté au travers d'une gamme particulière de comportements: les conduites de mauvaise foi qui sont, dit Sartre, un «art de former des concepts contradictoires $»^{3}$. Ainsi la réalisation émotive d'un possible renvoie en définitive à la concrétisation de la liberté au travers d'un comportement de mauvaise foi qui

${ }^{1}$ ETE, p. 91.

${ }^{2}$ ETE, p. 87.

${ }^{3} E N$, p. 91. 
ne conserve pas les normes et les critères de la vérité, comme ils sont acceptés par la pensée critique de bonne foi. Ce dont [...] décide [la mauvaise foi], en effet, d'abord, c'est de la nature de la vérité. Avec la mauvaise foi apparaît une vérité, une méthode de penser, un type d'être des objets; et ce monde de mauvaise foi, dont le sujet s'entoure soudain, a pour caractéristique ontologique que l'être y est ce qu'il n'est pas et n'y est pas ce qu'il est. En conséquence, un type d'évidences singulier apparaît : l'évidence non persuasive ${ }^{1}$.

Ainsi, en tant qu'elle est émotive, l'intentionnalité est à la fois une réponse, prenant la forme d'une négation protensive et intransitive $\left(1^{\circ}\right)$; un projet de transformation, constituant le monde selon une sémantique réglée par la magie $\left(2^{\circ}\right)$; et une conduite passionnelle manifestant, selon J. Englebert, une 《liberté qui s'adapte $»^{2}$ aux difficultés par une conduite de mauvaise foi $\left(3^{\circ}\right)$. Loin d'être un simple jeu de reformulations, cette polysémie de l'intentionnalité émotive permet de mettre en évidence les différents niveaux de complexité de la théorie des émotions. Lorsqu'il examine les caractéristiques de l'intentionnalité émotive qui ont trait à sa puissance de négation, l'ouvrage de 1939 présente d'abord un état de conscience particulier. Sartre décrit en effet la conscience « émue» en focalisant l'attention sur un mode intentionnel parmi d'autres que la conscience peut adopter. Par un examen de différentes situations pathologiques, il détaille ensuite les propriétés du projet émotif. Il s'agit alors pour l'Esquisse de mettre en évidence les processus concrets corrélés aux états émotifs de la conscience. Après quoi, L'Être et le Néant approfondit les remarques de 1939 dans une analyse des conduites passionnelles. Et cette reprise débouche sur une étude plus générale de la dimension affective de l'existence, dans la mesure où l'Essai de 1943 épingle la passion comme une forme d'expression toujours possible de la liberté. Enfin, il nous semble que la théorie des émotions culmine dans une étude rigoureuse de la mauvaise foi où Sartre dégage les propriétés ontologiques qui sont celles des objets visés dans une émotion, mais aussi, et surtout, «l'évidence non-persuasive» comme une forme de rationalité proprement émotive.

Néanmoins, qu'elle soit une puissance, un projet ou une conduite, en tant qu'elle est d'abord et avant tout une intentionnalité, l'émotion et la théorie dont elle fait l'objet renvoient encore et toujours à ce même lieu

\footnotetext{
${ }^{1} E N$, p. 103.

2 J. Englebert, « La magie et la sorcellerie », art. cit., p. 59.
} 
nocturne ${ }^{1}$ de la conscience qui est son néant interne. Or, puisque l'acquis historique et incontournable de L'Être et le Néant est d'avoir montré que « la liberté coïncide en son fond avec le néant qui est au cœur de l'homme $»^{2}$, les fondations de la théorie des émotions résident, en définitive, dans ce que R. Gély désigne comme la «puissance inouïe d'une liberté » ${ }^{3}$ dont les intentionnalités émotives ne sont rien moins que la plus brutale manifestation.

\section{L'émotion comme passion active}

Bien que les analyses menées plus haut aient permis d'identifier la complexité ainsi que la cohérence intrinsèque de la théorie des émotions, une difficulté subsiste. Sartre décrit l'intentionnalité émotive comme une conduite ineffective, mais soutient également que la conduite passionnelle est une manière d'agir dans le monde ${ }^{4}$. Si bien que, selon H. Oulc'hen, l'Esquisse semble affirmer que la passion est, sinon une action à part entière, du moins une "forme d'action continuée ${ }^{5}$. Ce qui est sans doute une ambiguïté fondamentale de l'ouvrage de 1939 car, comme le remarque V. de Coorebyter à la suite d'une remarque de D. Giovannangeli, en s'interdisant de « distinguer entre l'action et la passion » ${ }^{6}$, Sartre aurait peut-être écrit une «théorie de l'émotion sans émotion ${ }^{7}$. Il nous semble à nouveau que cette difficulté est moins une incohérence de l'Esquisse que l'un de ses principaux enjeux. En repartant d'une description des conduites volontaires du pour-soi, nous allons montrer que, dès 1939, Sartre a non seulement doté l'émotion de son propre pouvoir d'activité mais a, plus encore, offert un nouveau départ à la catégorie de l'action elle-même.

Puisqu'une action est «par principe intentionnelle ${ }^{8}$, la polysémie de l'intentionnalité autorise que l'on décrive l'action, à l'instar de l'émotion,

\footnotetext{
${ }^{1} C f$. J. Bourgeault, « Névrose et fiction herméneutique. Quelques remarques sur la méthode de L'Idiot de la famille », dans C. Lanzmann (dir.) Les Temps Modernes $\mathrm{n}^{\mathrm{o}} 667$ : Lecteurs de Sartre, Paris, Gallimard, 2012, p. 68-69 ; cf. R. Gély, «Émotion, imagination, incarnation », art. cit., p. 343.

${ }^{2} E N$, p. 485.

${ }^{3}$ R. Gély, «Émotion, imagination, incarnation », art. cit., p. 327.

${ }^{4}$ ETE, p. $79 \& 81$.

${ }^{5} \mathrm{H}$. Oulc'hen, «L'émotion dans la pratique », art. cit., p. 171.

${ }^{6}$ D. Giovannangeli, Le Retard de la conscience, Husserl, Sartre, Derrida, Bruxelles, Ousia, 2001, p. 62.

${ }^{7}$ V. de Coorebyter, « Le corps et l'aporie du cynisme », art. cit., p. 274.

${ }^{8} E N$, p. 477.
} 
comme une puissance $\left(1^{\circ}\right)$, un projet $\left(2^{\circ}\right)$ et une conduite $\left(3^{\circ}\right) .1^{\circ}$ Pour Sartre, l'action désigne une double puissance de négation. Car, lorsqu'il agit, le pour-soi « pose un état de choses idéal comme pur néant présent» de même qu'il «pose la situation actuelle comme néant par rapport à cet état de choses ${ }^{1}$ possible. $2^{\circ}$ Mais l'action est aussi un projet de modification dans la mesure où le pour-soi transite d'une situation réelle à une situation possible en agençant des «moyens en vue d'une fin $»^{2} .3^{\circ} \mathrm{Si}$ bien que, dans l'Esquisse, Sartre peut décrire l'action comme une « intuition pragmatique »" et, dans L'Etre et le Néant, comme une conduite volontaire qui

envisagera techniquement la situation, refusera le magique et s'appliquera à saisir les séries déterminées et les complexes instrumentaux qui permettent de résoudre des problèmes. Elle organisera un système de moyens en se basant sur le déterminisme instrumental. Du coup, elle découvrira un monde technique, c'est-à-dire un monde dans lequel chaque complexe-ustensile renvoie à un autre complexe plus large et ainsi de suite ${ }^{4}$.

Pour le dire vite, l'action « constitue une certaine couche existentielle dans le monde $»^{5}$ dans la mesure où cette double négation transitive modifie effectivement la 《 figure $»^{6}$ de la situation en y instituant des dispositifs instrumentaux, interconnectés selon les lois du déterminisme causal. Mais en élaborant des chaines d'ustensiles, l'action rend aussi le monde plus complexe et plus difficile à vivre. La notion de "difficulté " imposée par le monde, qui est le détonateur de l'émotion, renvoie ainsi à l'action. La difficulté à l'origine de l'intentionnalité émotive semble même "le corrélatif noématique de notre activité entreprise ou simplement conçue ${ }^{7}$. Donc tout se passe comme si la conduite volontaire contenait virtuellement une émotion, bien que Sartre ne l'affirme jamais comme tel. Bien plus, avec H. Oulc'hen on peut comprendre l'intentionnalité émotive comme «une

\footnotetext{
${ }^{1} E N$, p. 479.

${ }^{2} I d$.

${ }^{3}$ ETE, p. 77.

${ }^{4} E N$, p. 489

${ }^{5}$ ETE, p. 76.

${ }^{6} E N$, p. 477.

${ }^{7}$ ETE, p. 78.
} 
continuation de l'action par d'autres moyens ${ }^{1}$, dans la mesure où le point d'arrivée de l'action coïncide avec le point de départ de l'émotion.

En conséquence de quoi, il apparaît que l'intentionnalité émotive comporte ses propres formes d'activité. En effet, en succédant à une conduite volontaire qui modifie l'état d'une situation, l'émotion assure un pouvoir de transformation de cette même situation. Cette transformation coïncide, ainsi que nous l'avons montré plus haut, avec une constitution magique de l'état de choses réel. Si bien que l'on peut voir maintenant que " le rôle de l'émotion, son action, ce en quoi elle agit et a prise » consiste, selon J. Englebert, en "l'introduction du "magique" dans le "réel" »". Et c'est précisément parce que «le réel et le magique sont toujours intriqués $»^{3}$ dans l'attitude émotive, que l'émotion peut être comprise comme un pouvoir d'adaptation à une situation-problème. Car en exagérant les structures potentielles d'une situation problématique, elle dissout la contrainte imposée par l'en-soi. Ce pouvoir d'adaptation s'apparente à un pouvoir de la mauvaise foi, dans la mesure où, d'exagérations en exagérations, la conscience s'arrange d'une situation-problème en supprimant le rapport qui l'y lie ; c'est-à-dire en s'en détournant purement et simplement, après s'être convaincue de l'inanité de cette situation à force d'évidences non persuasives et de concepts contradictoires. Cela fait de l'émotion un art de l'esquive ; mais aussi, et surtout, un pouvoir de neutralisation de l'engagement reliant la conscience à son monde. En même temps qu'elle contourne les difficultés du monde réel, la conscience émue "chute ${ }^{4}$ dans un monde magique dont les structures ont été imaginées de telle manière qu'elles assouvissent immédiatement les désirs du pour-soi. Si bien que, semblable à une « conscience qui s'endort » ${ }^{5}$, ce dernier cesse de réitérer son projet dans une action mondaine. Faute de pouvoir faire exister ses possibles au travers d'elle, le pour-soi, si l'on en croit les analyses de R. Gély, «entretient l'illusion d'un engagement » dans un monde magique auquel la mauvaise foi lui donne le moyen de croire. Cependant, sous un autre angle, ce pouvoir de neutralisation émotif peut être également compris comme un pouvoir d'amplification de l'engagement reliant la conscience à son monde; dans la mesure où, après avoir ouvert la

\footnotetext{
${ }^{1}$ H. Oulc'hen, "L'émotion dans la pratique», art. cit., p. 178. Réciproquement, $\mathrm{H}$. Oulc'hen décrit l'action comme « la seule pierre de touche qui nous permette de comprendre l'émotion » (ibid, p. 172).

${ }^{2}$ J. Englebert, « La magie et la sorcellerie des visages », art. cit., p. 47.

${ }^{3}$ Ibid., p. 48.

${ }^{4}$ ETE, p. 115.

${ }^{5}$ ETE, p. 99.

${ }^{6}$ R. Gély, «Émotion, imagination, incarnation », art. cit., p. 329.
} 
conscience à des possibles inédits, la conduite passionnelle découvre ses propres «chemins de la liberté ${ }^{1}$ au lieu de suivre ceux suggérés par la situation ou par autrui. C'est pourquoi, enfin, l'intentionnalité émotive est un pouvoir de renouvellement en ce qu'elle permet à la conscience de scénariser, et même de théâtraliser à nouveaux frais, sa vie intentionnelle de telle manière que, si elle ne s'en estimait satisfaite, la conscience retrouve «son pouvoir primitif de recommencer $»^{2}$.

Ainsi, l'émotion peut être décrite comme un pouvoir de transformation du sens, d'adaptation à la difficulté, de neutralisation de l'action mondaine, d'amplification de l'engagement et, enfin, comme un pouvoir de renouvellement de la liberté. En refusant d'analyser l'émotion comme un état passif d'une conscience séparée de l'action, au lieu de poser une frontière qui sépare la passivité de l'activité, l'Esquisse d'une théorie des émotions s'est donc employée à concevoir l'intentionnalité émotive comme ce qu'il convient d'appeler une passion active poursuivant l'action au travers d'autres formes d'activité. C'est pourquoi, selon G. Cormann, tout porte à croire que «l'émotion est chez Sartre une pièce essentielle d'une théorie de l'action ${ }^{3}$. En ce sens, l'ouvrage de 1939 permet de renouveler significativement l'intelligibilité de la «catégorie cardinale de l'action»" ${ }^{4}$. Car au lieu que son extension se limite aux conduites volontaires, l'action désigne l'ensemble formé par l'addition des conduites volontaires et des passions actives. Maintenant, nous allons voir que leurs pouvoirs assurent une fonction cruciale dans l'économie globale de la conscience, à savoir la stabilisation de son ipséité.

\section{4. Émotivité et singularité}

Constitué par un projet intentionnel qui relie la conscience à ses potentialités ${ }^{5}$, le circuit de l'ipséité peut être, de manière plus précise, décomposé en trois aspects : la "facticité " ${ }^{6}$, qui désigne l'ensemble des déterminations contingentes de l'être pour-soi ; le possible projeté, vers lequel la conscience converge asymptotiquement; et le monde, qui est «l'obstacle nécessaire » ${ }^{7}$

\footnotetext{
${ }^{1}$ R. Gély, « Émotion, imagination, incarnation », art. cit., p. 410.

${ }^{2}$ Ibid., p. 344.

${ }^{3}$ G. Cormann, «Émotion et réalité chez Sartre, art. cit., p. 296.

${ }^{4} E N$, p. 477.

${ }^{5} E N$, p. 234

${ }^{6} E N$, p. 119.

${ }^{7}$ EN, p. 141.
} 
traversé par le pour-soi lorsqu'il réalise ses possibles en dépassant la figure de sa facticité. La singularité du pour-soi est ainsi une tension répartie sur ces trois termes. Par conséquent, son ipséité doit être pensée sur le mode d'un équilibre dynamique, sinon rompu, du moins déséquilibré par chacun des termes qu'il relie. Quant à l'émotion, on va voir qu'elle peut être systématiquement comprise comme un mécanisme de stabilisation des tensions parcourant ce circuit.

Puisqu'il désigne l'obstacle nécessaire par lequel la conscience doit transiter pour parvenir à ses fins, le monde est sans doute le lieu de tous les périls de l'ipséité. Car, en opposant un coefficient d'adversité trop élevé, le monde peut condamner la voie qui conduit la conscience à ses possibles et, de ce fait, pulvériser son ipséité qui se heurte de plein fouet à l'en-soi transphénoménal. Le pouvoir de neutralisation propre à l'intentionnalité émotive vise donc à empêcher une surtension de difficultés qui pourrait aboutir à un court-circuit de l'ipséité. Car l'émotion assure une transformation magique qui diminue le coefficient d'adversité opposé par l'être-en-soi transphénoménal à l'ipséité de l'être-pour-soi. L'émotion harmonise, en d'autres mots, l'ipséité et la situation où ce circuit se ramifie. Ainsi, même si la conduite passionnelle donne toutes les apparences d'une «fuite sur place $»^{1}$ lorsqu'elle fait en sorte que «l'univers n'exige plus rien de nous $»^{2}$, il n'en demeure pas moins que cette transformation magique est une manière de préserver l'équilibre identitaire du pour-soi en détournant le sens d'une situation qui pourrait mettre à mal son ipséité.

La facticité du pour-soi est elle aussi une source constante de déséquilibre identitaire. Pour être soi-même, il faut que le pour-soi intègre l'ensemble des déterminations sociales et biographiques qui esquisse les grandes lignes de sa condition individuelle, mais aussi des tensions à canaliser dans une structure identitaire. Là encore, c'est tout un usage de l'émotion qui semble être impliqué, dans la mesure où la conduite passionnelle permet d'intégrer les déterminations facticielles. Intégrer un rôle socialement défini, ou même seulement assumer l'héritage d'une condition physique, sont des actions qui supposent, d'une part, la capacité de s'adapter continuellement à toutes les formes de déterminations; et, d'autre part, un pouvoir de «théâtralisation $»^{3}$ de ces déterminations de telle manière qu'elles puissent être mises en scène, subverties et donc reprises dans un projet visant de nouvelles situations. Or, affirment J. Englebert et G.

${ }^{1}$ R. Gély, «Émotion, imagination, incarnation », art. cit., p. 313.

${ }^{2}$ ETE, p. 87.

${ }^{3}$ R. Gély, « Émotion, imagination, incarnation », art. cit., p. 313. 
Cormann, l'émotion «produit grâce à sa force magique [...] le dépassement d'une situation ${ }^{1}$ facticielle initiale. En d'autres termes, cette variété d'intentionnalité rend la conscience capable d'intégrer des évènements et des déterminations facticielles dans un projet personnalisant. Elle nivèle les caractéristiques contingentes de la conscience dans une architecture plus complexe qui est celle de son circuit de l'ipséité : en somme, elle assimile une diversité de déterminations contingentes dans une identité. Et elle donne, en outre, une « clé permettant de comprendre [...] ce qu'un homme parvient à faire de ce qu'on a fait de lui $»^{2}$. Car tout semble indiquer que l'émotion est le travail médiateur rendant intelligible l'intégration de différentes déterminations extérieures dans un même projet identitaire qui définit la singularité d'un homme.

Enfin, il faut remarquer que l'émotion contribue à stabiliser le projet d'un possible et maintient, par là même, l'équilibre de son ipséité. En effet, dès l'instant où le pour-soi projette un possible, une émotion doit pouvoir s'activer implicitement pour maximaliser la tension du circuit de l'ipséité. Car poser un possible c'est, pour ainsi dire, faire le pari qu'il pourra être réalisé. En d'autres termes, dès lors que la conscience s'élance vers une fin, il faut qu'elle nourrisse, au travers d'une évidence non persuasive, au travers de concepts contradictoires, l'illusion présomptive d'un engagement dans le monde. Il faut, comme l'indiquaient déjà les Carnets de la drôle de guerre, que le pour-soi use d'un pouvoir qui le plonge tout entier dans un « réconfort magique $»^{3}$ qui permettra, ensuite, "d'articuler, grâce à une série de récits et de pratiques, sa situation à d'autres situations ${ }^{4}{ }^{4}$ jusqu'à ce qu'il parvienne à son possible. Et ce n'est qu'une fois atteint cet état de «confiance magique $»^{5}$ qu'une « conscience parfaitement irréelle ${ }^{6}$ se risquera à devenir dans le monde la singularité qu'elle veut être, grâce aux transformations opérées par les passions actives; si ce n'est par le biais des mécanismes de persuasion propres à la mauvaise foi qui ne sont rien moins qu'une forme exacerbée de magie.

\footnotetext{
${ }^{1}$ G. Cormann \& J. Englebert, « Des situations-limites au dépassement de la situation : phénoménologie d'un concept sartrien », Sartre Studies International, vol. 22, 2016.

${ }^{2} I d$.

${ }^{3}$ J.-P. Sartre, Carnets de la drôle de guerre, dans Les Mots et autres écrits autobiographiques, Paris, Gallimard, « Bibliothèque de la Pléiade », 2010, p. 346.

${ }_{5}^{4}$ R. Gély, «Émotion, imagination, incarnation », art. cit., p. 406.

${ }^{5}$ J.-P. Sartre, Carnets de la drôle de guerre, op. cit., p. 355.

${ }^{6}$ J.-P. Sartre, L'Idiot de la famille. Gustave Flaubert de 1821 à 1857, Paris, Gallimard, 1988, p. 655.
} 
Ainsi, parce qu'elle neutralise les difficultés du monde, transforme la facticité, dépasse les situations, la passion assure une fonction nécessaire à l'économie générale de la conscience : elle stabilise le circuit identitaire du pour-soi en équilibrant, par des processus magiques, ses différents lieux de tensions. En sorte qu'à même l'émotivité nous trouvons un préalable à la singularité. Néanmoins, nous ne pourrions conclure cette analyse sans rappeler le caractère temporaire de cet équilibre. À trop séjourner dans ce que A. Hervy désigne comme «l'état d'une recréation du monde ${ }^{1}$, inauguré par les transformations magiques du projet émotif, la conscience est en effet 《victime de son propre piège $»^{2}$. Dans la mesure où «elle vit le monde nouveau qu'elle vient de constituer », elle prend un peu trop immédiatement ses désirs pour des réalités ; bref, « elle souffre les qualités que les conduites [passionnelles] ont élaborées $»^{3}$. Les émotions « fonctionnent à vide $»^{4}$, dit $\mathrm{R}$. Breeur, et elles risquent dès lors de déstabiliser la conscience puisque les qualités magiques, auxquelles elle croit dur comme fer, se font de plus en plus écrasantes ${ }^{5}$. Toutefois, il n'en reste pas moins que la passion active soutient de bout en bout la conscience. Car elle préserve son équilibre identitaire, signifiant ainsi, en dépit de son caractère métastable, « le tout de la conscience ou, si nous nous plaçons sur le plan existentiel, de la réalitéhumaine $»^{6}$. En effet, dans l'émotion, nous n'apercevons rien moins qu'une 《réalisation d'essence de la liberté-humaine en tant qu'elle est affection ${ }^{7}$; nous n'observerons rien d'autre, pour le dire autrement, qu'une existence personnelle se faisant sous les auspices d'une libre passion magique.

Fondées principalement sur un dialogue entre l'Esquisse d'une théorie des émotions et L'Être et le Néant, les analyses que l'on a formulées ont permis de clarifier la polysémie du concept d'intentionnalité émotive. De cet examen, il ressort que «l'émotion est une forme organisée de l'existence humaine $»^{8}$. Sa condition de possibilité réside dans le néant interne du poursoi dont elle est l'expression au travers d'une puissance de négation intran-

${ }^{1}$ A. Hervy, «Émotion et aliénation », art. cit., p. 30.

${ }^{2}$ ETE, p. 101.

${ }^{3}$ ETE, p. 98.

${ }^{4}$ R. Breeur, « Passion à volonté », art. cit., p. 17.

${ }^{5}$ ETE, p. 104.

${ }^{6}$ ETE, p. 26.

${ }^{7}$ ETE, p. 124.

${ }^{8}$ ETE, p. 26. 
sitive, d'un projet de transformation magique du monde et d'une conduite passionnelle produisant un assouvissement symbolique des désirs du poursoi. Son apparition survient lorsque l'en-soi fait opposition aux projets de la conscience. Son pouvoir d'action, justifiant que l'on rebaptise l'émotion du nom de "passion active », consiste à neutraliser cette difficulté ; c'est-à-dire à adapter une facticité, à dépasser une situation, à transformer le monde, à renouveler la conscience, bref, à stabiliser son ipséité en amplifiant ou en réduisant les tensions de ce circuit. Sa nécessité nait de ce qu'elle est dotée de ses propres formes d'activité qui permettent à la conscience d'agir sur le sens de sa situation, d'être lestée d'une structure identitaire, et de trouver ses propres chemins de libération en posant des possibles inédits. Cela revient à dire que l'émotion est nécessaire dans la mesure où, comme l'a remarqué R. Gély, elle fait en sorte que « la liberté retrouve les sources primitives de sa puissance $»^{1}$. Ses enjeux philosophiques, enfin, sont multiples.

L'Esquisse d'une théorie des émotions renseigne, en premier lieu, sur la nature du phénomène émotif qui est décrit à la fois comme un état de conscience, un processus actif et une dimension affective de l'existence. En appliquant toutes sortes de torsions au concept d'intentionnalité, Sartre infléchit durablement la phénoménologie dans la direction d'un traité des passions grâce auquel nous apprenons à mieux connaître les émotions dont nous sommes le sujet. En second lieu, l'ouvrage de 1939 refonde la signification du concept d'action qui embrasse à la fois les conduites volontaires et les passions actives. Cela met sérieusement à mal une tendance "assez commune $»^{2}$ et pour le moins vulgaire qui assimile les actes libres aux actes volontaires. Tout au contraire, Sartre apprend que nous sommes libres partout, jusque dans l'insomnie, les pleurs et les rires ; jusque dans la jalousie et l'angoisse de mourir qui est pire que tout cela, disait Proust dont l'Esquisse invite à refuser les analyses intellectualistes de la passion. Enfin, en troisième lieu, en plus de penser d'une manière originale l'émotion comme une activité, l'Esquisse, relue à partir de L'Être et le Néant, permet de concevoir l'interactivité existant entre la passion active et le circuit de l'ipséité. Ainsi l'originalité de la théorie des émotions s'approfondit dans une théorie de l'identité personnelle où l'on découvre que la passion est un régulateur de tension du circuit de l'ipséité et, plus globalement, que l'émotion est condition de la singularité de la conscience.

Plus largement encore, les analyses menées plus haut indiquent que l'intentionnalité peut être un fil conducteur solide pour dégager la cohérence

${ }^{1}$ R. Gély, « Émotion, imagination, incarnation », art. cit., p. 248.

${ }^{2} E N$, p. 485 . 
de la production philosophique du premier Sartre. Car, «malgré et grâce à l'impulsion décisive du concept d'intentionnalité ${ }^{1}$, ce dernier aurait, d'après V. de Coorebyter, non seulement «réinventé la pratique phénoménologique $»^{2}$ mais aurait, en outre, réalisé ce geste déterminant qui consiste à "inscrire l'intentionnalité affective dans le parcours personnel du sujet $\rangle^{3}$. Cela justifie, d'après nous, le gain qu'il peut y avoir à lire à rebours l'Esquisse à partir de l'essai d'ontologie phénoménologique qui développe l'idée d'un circuit de l'ipséité, plutôt qu'avec La Transcendance de l'Ego dont la conclusion interdit pour ainsi dire au phénoménologue d'attribuer une personnalité à la conscience ${ }^{4}$.

Enfin nous espérons que notre travail permettra d'un peu mieux comprendre en quel sens « le monde social est », selon Sartre, «magique » ${ }^{5}$. Au terme de notre analyse de l'intentionnalité émotive, nous avons en effet dégagé différentes formes d'activité propre à l'émotion. La passion active nous est ainsi apparue comme une continuation de la conduite volontaire, et nous avons dès lors cessé de placer une frontière séparant l'activité de la passivité de la conscience. Mais l'absence de cette cloison fait voir que l'activité du pour-soi dans le monde, ainsi que celle des pour-soi entre eux, réfère aussi aux projets émotifs par lesquels les consciences entreprennent la saisie de rapports nouveaux, c'est-à-dire aux transformations au travers desquelles elles étoffent le monde d'une dimension magique. Aussi l'enjeu de l'abolition d'une telle frontière entre passivité et activité coïncide-t-il avec l'élaboration d'une catégorie de l'action renouvelée comprenant à la fois les conduites volontaires et les passions actives, induisant du même coup une dialectisation de ces deux modalités de la liberté du pour-soi, mais cernant aussi quelque chose de l'ordre d'une économie passionnelle en creux des rapports actifs que les consciences nouent entre elles. En ce sens, les rapports d'interdépendances entre les pour-soi comporteraient une dimension magique qui est pour ainsi dire le résultat de cette économie passionnelle. Toutefois, si nous avons pourtant renoncé à analyser de manière plus approfondie cette dimension magique, dimension dont Sartre thématise l'apparition comme une seconde forme d'émotion ${ }^{6}$, c'est que nous avons constaté que l'ampleur de

\footnotetext{
${ }^{1}$ V. de Coorebyter, Sartre face à la phénoménologie, op. cit., p. 168. ${ }^{2} I d$.

${ }^{3}$ V. de Coorebyter, Sartre face à la phénoménologie, op. cit., p. 131.

${ }^{4}$ Après avoir identifié la personne à l'Ego et ce dernier à un objet transcendant par rapport à la conscience, Sartre conclut en effet que la « conscience transcendantale est une spontanéité impersonnelle » (TE, p. 79).

${ }^{5}$ ETE, p. 108.

${ }^{6}$ ETE, p. 109.
} 
cette tâche commanderait de dépasser les jalons qui ont été posés ici. Car, comme nous l'avons appris d'Alievtina Hervy, comprendre en son fond la magie originaire du social supposerait de tirer au clair le phénomène d'aliénation de la conscience ${ }^{1}$. Cela impliquerait également d'embrayer la phénoménologie de l'affectivité de 1939 à une "philosophie de la praxis ${ }^{2}$ qui permettrait, si nos présomptions sont exactes, de dégager avec $\mathrm{H}$. Oulc'hen la structure " pratico-magique $»^{3}$ du pratico-inerte. Dans l'attente d'un tel exercice, nous pouvons lire et réfléchir une théorie raffinée de l'intentionnalité émotive où l'on apprend que l'émotion, "ordinairement tenue pour un désordre sans loi » ${ }^{4}$, est une passion active à l'endroit de la personne qui n'est rien d'autre que la "signification humaine de la liberté » ${ }^{5}$; rien de moins, écrit Sartre, que la "vérité de la liberté ${ }^{6}$, où trouve sa fondation le discours tenu dans L'Esquisse d'une théorie des émotions.

\footnotetext{
${ }^{1}$ A. Hervy, « Émotion et aliénation », art. cit., p. 35.

${ }^{2}$ G. Cormann, «Passion et liberté », art. cit., p. 95.

${ }^{3} \mathrm{H}$. Oulc'hen, «L'émotion dans la pratique », art. cit., p. 183.

${ }_{5}^{4}$ ETE, p. 122.

${ }^{5} E N$, p. 613.

${ }^{6} I d$.
} 